\section{Type 2 Diabetes and COVID-19- Related Mortality in the Critical Care Setting: A National Cohort Study in England, March-July 2020}

Diabetes Care 2021;44:50-57 | https://doi.org/10.2337/dc20-1444

\section{OBJECTIVE}

To describe the relationship between type 2 diabetes and all-cause mortality among adults with coronavirus disease 2019 (COVID-19) in the critical care setting.

\section{RESEARCH DESIGN AND METHODS}

This was a nationwide retrospective cohort study in people admitted to hospital in England with COVID-19 requiring admission to a high dependency unit (HDU) or intensive care unit (ICU) between 1 March 2020 and 27 July 2020. Cox proportional hazards models were used to estimate 30-day in-hospital all-cause mortality associated with type 2 diabetes, with adjustment for age, sex, ethnicity, obesity, and other major comorbidities (chronic respiratory disease, asthma, chronic heart disease, hypertension, immunosuppression, chronic neurological disease, chronic renal disease, and chronic liver disease).

\section{RESULTS}

A total of 19,256 COVID-19-related HDU and ICU admissions were included in the primary analysis, including 13,809 HDU (mean age 70 years) and 5,447 ICU (mean age 58 years) admissions. Of those admitted, 3,524 (18.3\%) had type 2 diabetes and 5,077 (26.4\%) died during the study period. Patients with type 2 diabetes were at increased risk of death (adjusted hazard ratio [aHR] 1.23 [95\% $\mathrm{Cl} 1.14,1.32$ ]), and this result was consistent in HDU and ICU subsets. The relative mortality risk associated with type 2 diabetes decreased with higher age (age 18-49 years aHR 1.50 [95\% Cl 1.05, 2.15], age 50-64 years 1.29 [1.10, 1.51], and age $\geq 65$ years 1.18 $[1.09,1.29] ; P$ value for age-type 2 diabetes interaction $=0.002$ ).

\section{CONCLUSIONS}

Type 2 diabetes may be an independent prognostic factor for survival in people with severe COVID-19 requiring critical care treatment, and in this setting the risk increase associated with type 2 diabetes is greatest in younger people.

In early 2020, coronavirus disease 2019 (COVID-19), caused by the new highly infectious organism now known as severe acute respiratory syndrome coronavirus 2 (SARS-CoV-2), was formally declared a public health emergency of international concern by the World Health Organization (1). With a global prevalence of $9.3 \%$ (2), it is unsurprising that diabetes is one of the most common comorbidities seen in people
John M. Dennis, ${ }^{1}$ Bilal A. Mateen, ${ }^{2,3}$ Raphael Sonabend, ${ }^{4}$ Nicholas J. Thomas, ${ }^{1,5}$ Kashyap A. Patel, ${ }^{1,5}$

Andrew T. Hattersley, ${ }^{1,5}$

Spiros Denaxas, ${ }^{2,6,7}$

Andrew P. McGovern, ${ }^{1,5}$ and

Sebastian J. Vollmer ${ }^{2,8}$
${ }^{1}$ Institute of Biomedical \& Clinical Science, University of Exeter Medical School, Exeter, U.K.

${ }^{2}$ The Alan Turing Institute, London, U.K.

${ }^{3}$ Social Science and Systems in Health Unit, Warwick Medical School, University of Warwick, Coventry, U.K.

${ }^{4}$ Department of Statistical Science, University College London, London, U.K.

${ }^{5}$ Royal Devon and Exeter National Health Service Foundation Trust, Exeter, U.K.

${ }^{6}$ Institute of Health Informatics, University College London, London, U.K.

${ }^{7}$ Health Data Research U.K., London, U.K.

${ }^{8}$ Department of Statistics, University of Warwick, Coventry, U.K.

Corresponding author: Bilal A. Mateen, bilal .mateen@nhs.net

Received 12 June 2020 and accepted 30 September 2020

This article contains supplementary material online at https://doi.org/10.2337/figshare.13034210.

This article is part of a special article collection available at https://care.diabetesjournals.org/ collection/diabetes-and-COVID19.

(C) 2020 by the American Diabetes Association. Readers may use this article as long as the work is properly cited, the use is educational and not for profit, and the work is not altered. More information is available at https://www.diabetesjournals .org/content/license. 
with COVID-19, second only to hypertension (3). People with type 2 diabetes are known to not only be more susceptible to infections in general (4) but also require hospitalization more often (5), resulting in an overall worse prognosis (6). As the COVID-19 pandemic has progressed, a similar pattern of a worse prognosis for people with diabetes who contract COVID-19 has been reported in population-based studies $(7,8)$, with an increased risk of intensive care admission (9-11) and worse mortality outcome in some but not all hospital cohorts (12-14).

For people admitted to intensive care, studies published early in the course of the global pandemic suggested diabetes is an important prognostic factor even in severe COVID-19 (New York, $n=373$, $40.2 \%$ with diabetes, unadjusted odds ratio [OR] for mortality in those with diabetes 2.31 [95\% Cl 1.34, 4.00], and Wuhan, China, $n=193,24.9 \%$ with diabetes, adjusted hazard ratio [aHR] 1.53 [95\% Cl 1.02, 2.30]) (11,15). Two larger, more recent critical care studies have reported smaller effect sizes (Italy, $n=$ $3,988,12.9 \%$ with diabetes, adjusted OR 1.18 [95\% Cl 1.01, 1.39], and U.S., $n=$ 2,215, 38.9\% with diabetes, adjusted OR 1.14 [95\% Cl 0.91, 1.43]) $(16,17)$. Heterogeneity across these studies likely reflects a combination of local variation in prevalence of diabetes, variation in the characteristics of people with diabetes, and the limited geo-temporal snapshots each study represents. Data on the additional mortality associated with diabetes in critical care across the entire first wave of COVID-19 are lacking in these studies, and potential heterogeneity in the risk associated with diabetes by age and other clinical features has yet to be fully explored.

We aimed to establish the clinical utility of type 2 diabetes as a prognostic factor for survival in people admitted to critical care with COVID-19, using national data over the course of the entire first wave of COVID-19 from hospitals in England.

\section{RESEARCH DESIGN AND METHODS}

\section{Data Source}

Data were accessed from the COVID-19 Hospitalisation in England Surveillance System (CHESS), established by Public Health England (PHE) (18). Reporting to CHESS is daily via Web tool and is mandatory for hospitals in England. The data specification comprises epidemiological information for people with proven or a high likelihood of COVID-19 requiring hospitalization and high dependency unit (HDU) or intensive care unit (ICU) management, including demographics, comorbidities including diabetes and obesity, and outcome. Confirmation of infection was by RT-PCR of nasopharyngeal and/or oropharyngeal swabs, as it was the only available method during the study period. National testing policy changed throughout the study period; however, all people with a suspicion of COVID-19 and who were admitted to hospital were tested (19), and people were potentially retested multiple times if the initial results were negative but clinical suspicion remained high. At the time of data extraction (27 July 2020), data on 23,935 cases across 108 hospitals had been submitted to CHESS.

\section{Study Population}

\section{Inclusion and Exclusion Criteria}

The study population comprised people in CHESS aged 18-99 years inclusive who required HDU or ICU admission with confirmed or clinically diagnosed COVID19 (91.4\% with PCR swab positive result at some point in their admission) from 1 March 2020 to 27 July 2020 inclusive and were not pregnant ( $n=22,082$ ). Pregnancy is associated with altered metabolic physiology; due to the limited information about this group, an a priori decision was made to exclude pregnant patients from the analysis.

\section{Cohorts}

The primary cohort for analysis was all patients admitted to either HDU or ICU at any point during the study period (HDU/ ICU cohort). We separately examined the subsets of all patients admitted to HDU but not ICU during the study period (HDU cohort) and all patients admitted to ICU during the study period (ICU cohort). People with type 1 diabetes were excluded from these primary cohorts and are analyzed separately.

\section{Recorded Clinical Features}

Available clinical features comprised demographic characteristics (age, sex, ethnicity), obesity status, and comorbidities, as defined in the CHESS data specification (18), recorded during critical care admission. We grouped ethnicity into major U.K. ethnic census groups: White, Black, Asian, mixed, other, or not recorded. We categorized obesity status as nonobese $\left(\mathrm{BMI}<30 \mathrm{~kg} / \mathrm{m}^{2}\right.$ ), obese (BMI $\geq 30 \mathrm{~kg} /$ $\mathrm{m}^{2}$ ), or not recorded, as derived from recorded BMI category (coded in CHESS as <18.5, 18.5-24.9, 25-29.9, 30-39.9, or $>39.9 \mathrm{~kg} / \mathrm{m}^{2}$ or no, missing, or unknown) where available or recorded clinical obesity (coded as no, borderline, yes, missing, or unknown) if BMI was not available. Coded comorbidities were diabetes (separated by type 1 and 2), chronic respiratory disease, asthma requiring medication, chronic/congenital heart disease, hypertension, immunosuppression (due to disease or treatment), chronic neurological disease, chronic renal disease, and chronic liver disease, all coded as no, yes, missing, or unknown.

\section{Missing Data}

We found variation in reporting of information on comorbidity at the hospital level, with a limited number of hospitals reporting very little information on comorbidity. For our primary analysis, we excluded all patients from 15 hospitals where comorbidity recording was missing for $>50 \%$ of people $(n=2,151)$ (Supplementary Flowchart 1). After filtering the CHESS cohort by missingness, we defined binary variables for each comorbidity (yes vs. no/missing/unknown), assuming that, in this mostly complete subset, missing or unknown coded data represented the absence of that condition. We tested the validity of this assumption in the sensitivity analysis, described below.

\section{Outcome}

The outcome was in-hospital all-cause mortality. People were followed up from admission to hospital until the earliest of the following: death, hospital transfer, date of last follow-up in CHESS, or a length of stay of 30 days. To account for discharge from hospital as a competing risk, we assumed that people who were discharged were no longer at risk of death, and so these individuals were not censored at the day of discharge and instead had their follow-up time set to 30 days (12).

\section{Statistical Analysis}

Primary Analysis

Recorded clinical features were described, overall and by recorded type 2 diabetes status. We estimated the risk of 30-day mortality for each cohort by type 2 diabetes status using survival analysis with days at risk as the timescale. Thirty-day 
mortality covered $98 \%$ of the deaths observed over the maximum cohort follow-up. Kaplan-Meier survival functions and Cox proportional hazards models were used to evaluate the association between type 2 diabetes and mortality, with proportional hazards assumptions tested through examination of the Schoenfeld residuals. All models included type 2 diabetes status and were sequentially increased in complexity with adjustment firstly for age (with exploration of age as linear, category, and a three-knot nonlinear restricted cubic spline), before addition of sex, ethnicity, obesity, and comorbidities. The primary, fully adjusted, model comprised all these features. For the ICU cohort, we started follow-up on the date of ICU admission and adjusted for days from hospitalization to ICU admission.

\section{Subgroup Analysis}

Analysis of the primary outcome for the HDU/ICU cohort was repeated in subgroups defined by major clinical characteristics; age (18-49, 50-64, $\geq 65$ years), sex, and ethnicity; the presence or absence of obesity, and the presence or absence of at least one other comorbidity. For exploration of temporal trends, subgroups were defined by calendar month of hospital admission (March, April, and May-July 2020).

\section{Sensitivity Analysis}

To explore the sensitivity to the decision to filter patients by degree of missing comorbidity data at hospital level, we described recorded characteristics of patients in included and excluded hospitals and assessed whether the mortality associated with type 2 diabetes differed in included and excluded hospitals. To explore sensitivity of our findings to potential temporal changes in mortality over the study period, calendar week of hospital admission was included as an additional linear continuous covariate in multivariable analysis.

We conducted a propensity scorematched analysis to evaluate whether the presence of type 2 diabetes increased the risk of death compared with risk in patients with the same clinical characteristics but without type 2 diabetes. The full set of recorded clinical features previously described was considered as potential confounders and used for matching. Missing information for ethnicity and obesity was coded as missing categories, providing a full patient set (20). Pairs were matched without replacement on the logit of the propensity score, and a caliper size of 0.05 was applied for all matched pairs. Separate propensity score models were fitted for each cohort. Cox proportional hazards models were stratified by matched set to account for the matched nature of the propensity score-defined cohorts.

We repeated the primary analysis with the following changes to formally assess the impact of the assumptions made: 1 ) excluding transferred patients and patients who remained in hospital, 2) including only patients with PCR swab positive results during admission, 3) including only hospitals with at least $75 \%$ complete comorbidity data, 4) including all patients in all hospitals and assuming that missing/unknown represented the absence of a comorbidity, 5) extending the Cox proportional hazards model to incorporate hospital-level random effects to account for within-hospital homogeneity in outcomes (frailty model with a random effect per hospital trust) (21), and 6) using a Fine and Gray model to check the validity of our approach by accounting for discharge as a competing risk.

\section{Evaluation of Missing Data}

To evaluate the appropriateness of equating diabetes status coded as missing in CHESS to the absence of diabetes, we used multiple imputation by chain equations (five imputations) to fill missing values for diabetes status and all other missing values for ethnicity, obesity, and other comorbidities. This approach assumes that missing values are missing at random (22). We then repeated the full multivariable analysis for each cohort using the imputation model, to determine whether this model gave different results to our primary analysis.

\section{COVID-19 Mortality Risk in People With \\ Type 1 Diabetes}

Mortality in people with recorded type 1 diabetes was compared with that in people with type 2 diabetes with adjusted Cox regression, as described above. A propensity-matched analysis was also used, as previously described, to compare the mortality risk for people with type 1 diabetes with that for similar patients without type 1 diabetes. Analyses were limited to the combined HDU/ICU cohort due to sample size.

\section{Computational Resources}

Analyses were conducted with $\mathrm{R}$ (version 3.6.2) (23), including use of the packages survival, rms, coxme, paf, mlr3, mlr3tuning, mlr3proba, xgboost, and mice.

\section{Ethics and Governance}

The study was reviewed and approved by the Warwick Biomedical \& Scientific Research Ethics Committee (BSREC 119/19$20)$, and sponsorship for this study was provided by University of Warwick (SOC. 28/19-20).

\section{Data and Resource Availability}

Data cannot be shared publicly, as it was collected by PHE as part of their statutory responsibilities, which allows them to process patient confidential data without explicit patient consent. Data used in this study were made available through an agreement between the University of Warwick and PHE. Individual requests for access to CHESS data are considered directly by PHE (contact via covid19surv@phe.gov.uk).

\section{RESULTS}

\section{Clinical Characteristics}

A total of 19,256 patients were included in the primary cohort admitted to HDU ( $n=13,809)$ or ICU $(n=5,447)$ (Supplementary Flowchart 1 and Supplementary Table 1). Mean age was 67 years, $60.1 \%$ of patients were male, $27.0 \%$ of patients were of non-White ethnicity, and $18.3 \%$ had type 2 diabetes. Supplementary Table 1 shows the overall characteristics of patients in the separate HDU and ICU cohorts. ICU patients were younger and more commonly male and of non-White ethnicity (mean age 58 years, $70.6 \%$ male, $37.1 \%$ non-White) than HDUonly admitted patients (mean age 70 years, $55.9 \%$ male, $23.1 \%$ non-White).

In the primary HDU/ICU cohort, patients with type 2 diabetes ( $n=3,524$ [18.3\%]) were of similar age to those without type 2 diabetes (mean 67 vs. 66 years, respectively) and were more commonly recorded as being of non-White ethnicity $(41.5 \%$ vs. $23.5 \%$ ) and obese ( $45.1 \%$ vs. $31.4 \%$ ) (Table 1). All comorbidities were more common in patients with type 2 diabetes.

Type 2 Diabetes Is an Independent Prognostic Factor for Mortality in Severe COVID-19

A total of 5,077 (26.4\%) patients in the primary HDU/ICU cohort died within the 30day study period. Mean (SD) follow-up was 21.2 (10.9) days in people with type 2 diabetes and 21.2 (19.4) days in those without. 
Table 1-Recorded characteristics of HDU/ICU cohort, by recorded type 2 diabetes status

\begin{tabular}{|c|c|c|}
\hline & $\begin{array}{c}\text { No type } 2 \text { diabetes } \\
(n=15,732)\end{array}$ & $\begin{array}{c}\text { Type } 2 \text { diabetes } \\
(n=3,524) \\
\end{array}$ \\
\hline Age (years) & $66(17.4)$ & $67(14.1)$ \\
\hline \multicolumn{3}{|l|}{ Age-group (years) } \\
\hline $18-24$ & $164(1.0)$ & $8(0.2)$ \\
\hline $25-34$ & 612 (3.9) & $40(1.1)$ \\
\hline $35-44$ & $1,010(6.4)$ & $142(4.0)$ \\
\hline $45-54$ & 2,039 (13.0) & $429(12.2)$ \\
\hline $55-64$ & $2,814(17.9)$ & $848(24.1)$ \\
\hline $65-74$ & $2,978(18.9)$ & $872(24.7)$ \\
\hline $75-84$ & $3,334(21.2)$ & $718(20.4)$ \\
\hline$\geq 85$ & $2,781(17.7)$ & $467(13.3)$ \\
\hline \multicolumn{3}{|l|}{ Sex } \\
\hline Female & $6,440(40.9)$ & $1,243(35.3)$ \\
\hline Male & $9,292(59.1)$ & $2,281(64.7)$ \\
\hline \multicolumn{3}{|l|}{ Ethnicity* } \\
\hline White & $9,624(76.5)$ & $1,815(58.5)$ \\
\hline Asian & $1,356(10.8)$ & $680(21.9)$ \\
\hline Black & 737 (5.9) & 360 (11.6) \\
\hline Mixed & $163(1.3)$ & $48(1.5)$ \\
\hline Other & $694(5.5)$ & $201(6.5)$ \\
\hline \multicolumn{3}{|l|}{ Obesity** } \\
\hline Nonobese & $6,034(68.6)$ & $1,509(54.9)$ \\
\hline Obese & $2,764(31.4)$ & $1,240(45.1)$ \\
\hline \multicolumn{3}{|l|}{ Comorbidity } \\
\hline Any comorbidity & 6,228 (39.6) & 2,945 (83.6) \\
\hline Chronic respiratory disease & $1,250(7.9)$ & $423(12.0)$ \\
\hline Asthma & $1,200(7.6)$ & $428(12.1)$ \\
\hline Hypertension & 3,439 (21.9) & $2,218(62.9)$ \\
\hline Chronic heart disease & $1,828(11.6)$ & $834(23.7)$ \\
\hline Chronic renal disease & $996(6.3)$ & $831(23.6)$ \\
\hline Chronic liver disease & $252(1.6)$ & $138(3.9)$ \\
\hline Chronic neurological disease & $1,098(7.0)$ & $310(8.8)$ \\
\hline Immunosuppressive disease & $374(2.4)$ & $94(2.7)$ \\
\hline
\end{tabular}

Data are $N(\%)$, except for Age (years), which is mean (SD). *Ethnicity not recorded for 3,578 patients. ${ }^{* *}$ Obesity not recorded for 7,709 patients.

Risk of mortality was consistently higher in people with type 2 diabetes in all analyses performed. The overall unadjusted cumulative incidence of 30-day mortality was higher in people with diabetes $134.7 \%$ [95\% Cl 33.1, 36.3]) than in people without diabetes (25.5\% [24.8, 26.2]) (Fig. 1). Mortality did not differ between people with diabetes recorded as missing/unknown diabetes status and those recorded as no diabetes (Supplementary Fig. 1), supporting the assumption that missing coding represents the absence of diabetes. Unadjusted hazard ratios and aHRs showed higher mortality risk in people with diabetes, with a $23 \%$ increase in mortality risk observed in the fully adjusted model (Table 2). Full multivariable model outputs are provided in Supplementary Table 2. A $23 \%$ increase in mortality risk for diabetes was also observed in the propensity scorematched analysis (Table 2).
The mortality increase in people with type 2 diabetes was consistent in the HDU cohort (type 2 diabetes 30-day mortality $29.2 \%$ [ $95 \% \mathrm{Cl} 27.2,31.1]$ vs. no diabetes $22.4 \%$ [21.6, 23.2]) and ICU cohort (30-day mortality $45.2 \%$ [42.4, 47.9] vs. 36.3\% [34.7, 37.8]) (Table 2 and Supplementary Fig. 2).

The mortality risk for type 1 diabetes ( $n=203$ ) was similar to that observed for type 2 diabetes (aHR 0.98 [95\% Cl 0.75, 1.28], $P=0.89$ ), and in propensity scorematched analysis the hazard ratio for type 1 diabetes was similar to that observed for matched control subjects without diabetes (aHR 1.25 [95\% Cl 0.86, 1.84], $P=0.25$ ).

\section{Subgroup Analysis Suggests Lower} Excess Mortality Risk Associated with Type 2 Diabetes at Older Ages and in Males Compared with Females

Results of subgroup analyses for the primary outcome are shown in Fig. 2. The association between type 2 diabetes and mortality was attenuated by age at hospital admission (age 18-49 years aHR 1.50 [95\% Cl 1.05, 2.15]), age 50-64 years $1.29[1.10,1.51]$, and age $\geq 65$ years 1.18 [1.09; 1.29$], P=0.002$ for age categorytype 2 diabetes interaction), and there was a numerically lower excess mortality risk in males $(1.15[1.05,1.26])$ than females (1.36 [1.20, 1.54], $P=0.06$ for sex-diabetes interaction). There was no evidence of a difference in the mortality associated with type 2 diabetes in subgroups defined by ethnicity $(P=0.08)$, obesity $(P=0.28)$, or presence or absence of other recorded comorbidity $(P=0.41)$. There was no evidence of a temporal trend in the mortality associated with type 2 diabetes by calendar month (hazard ratio range 1.131.34 over March-July) (Supplementary Table 3) or with adjustment for calendar week of hospital admission as an additional covariate (aHR for diabetes in the primary HDU/ICU cohort $1.21[95 \% \mathrm{Cl}$ 1.13, 1.31]) (Supplementary Table 3).

\section{Sensitivity and Multiple Imputation Analyses Were Consistent With the Primary Analysis}

Results were consistent in all sensitivity analyses (Supplementary Table 3), including inclusion of only patients with a positive PCR swab result during admission; repetition of the primary analysis with hospital added as a random effect in the multiple imputation model; and, despite differences in clinical characteristics (Supplementary Table 4), inclusion of patients from all hospitals currently reporting to $\mathrm{CHESS}$ irrespective of completeness of comorbidity coding.

\section{CONCLUSIONS}

Our analysis of $>19,000$ patients admitted to critical care over the entire first wave of COVID-19 in England demonstrates that type 2 diabetes is associated with $\sim 20 \%$ increase in mortality risk in people with severe COVID-19, independent of age, sex, ethnicity, obesity, and other major comorbidity. Risk is similar in both the HDU and ICU cohorts, despite the fact that in England these groups differed markedly, with those admitted to ICU being younger, more commonly male, and more commonly of non-White ethnicity. Additional mortality risk appears similar in type 1 diabetes compared with type 2 diabetes in this setting. 


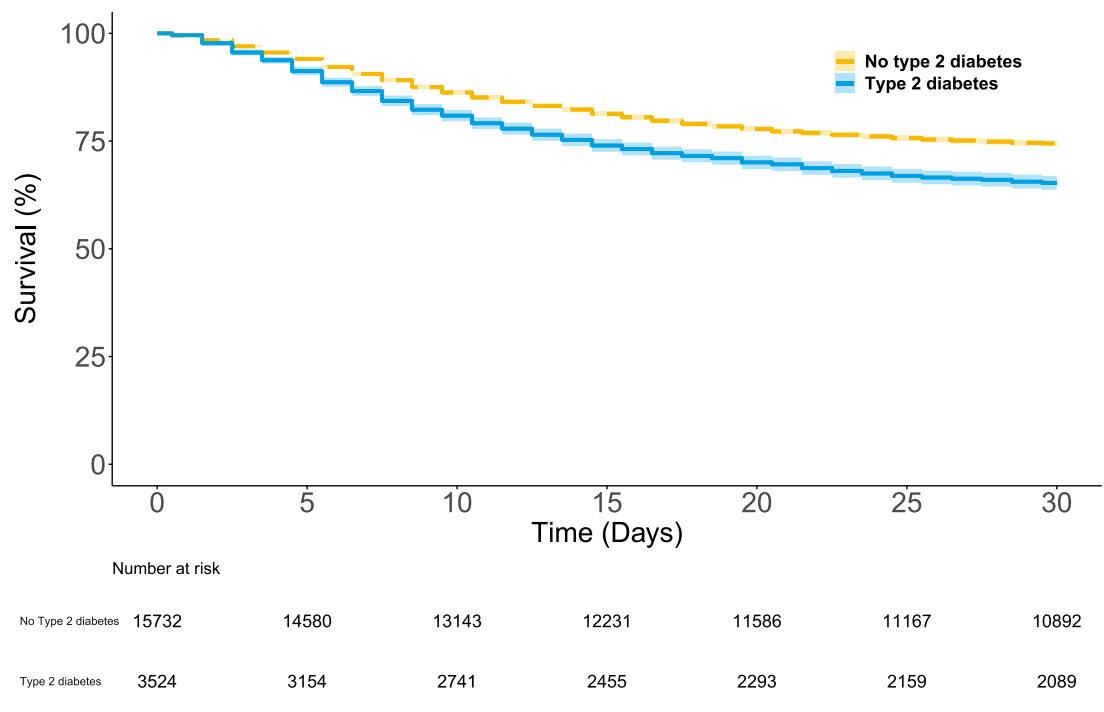

Figure 1-Kaplan-Meier plots for in-hospital COVID-19 death in 19,256 patients admitted to critical care (HDU or ICU) in CHESS by time since hospital admission. Plots show the proportion of individuals at risk who were still alive at regular intervals up to 30 days from admission, stratified by the presence of type 2 diabetes. People discharged from hospital prior to 30 days were assumed to survive to 30 days and are included in the number at risk until 30 days, in keeping with the standard practice for time-to-event modeling in analysis of critical care patients.

Our national-level study provides evidence for a relatively small, but significant, effect of diabetes on mortality risk in one of the largest samples of COVID19-associated critical care admissions to date. The association between diabetes and COVID-19 that mortality we observed is substantially smaller than those reported in studies conducted early in the pandemic $(11,15)$. Notably, we extend previous contributions by evaluating both HDU and ICU admissions, which we demonstrate to be very different populations in terms of clinical characteristics. In England, these differences between HDU and ICU populations likely result from individualized care strategies based on the patient's best interests (which one UKbased center implemented for $61 \%$ of 429 consecutive admissions) (24) rather than sustained saturation of ICUs, for which there was limited evidence (25).
Given the large sample available, we were able to evaluate heterogeneity in mortality risk across subgroups defined by clinical features and demonstrate that, in relative terms, the additional mortality risk associated with type $2 \mathrm{di}$ abetes attenuates markedly in older people. In contrast to age, we found no evidence of heterogeneity in the mortality risk associated with diabetes in subgroups defined by the presence or absence of obesity, comorbidity, and ethnicity. There was weak evidence of heterogeneity by sex, with a numerically higher excess risk associated with diabetes in females than males $(P=0.06)$, which may warrant future investigation. Importantly, we demonstrate a consistent strength of association between type 2 diabetes and mortality accounting for time trends in in-hospital mortality, and accounting for geographical clustering of hospitals, which is notable, as recent studies have demonstrated substantial variation in COVID-19-specific critical care outcomes between institutions (26), as well as a marked reduction in critical care mortality over the course of the pandemic (27).

Although direct evaluation of patient characteristics other than diabetes was not the focus of this study, the validity of our results is bolstered by demographic congruence with COVID-19 data from the U.K.'s Intensive Care National Audit \& Research Centre (ICNARC) (28), where diabetes status is not available. Notably, both studies found similar effects for

Table 2-Hazard ratios for diabetes for the primary outcome of $\mathbf{3 0}$ day in-hospital mortality for people with type 2 diabetes admitted to critical care with COVID-19

\begin{tabular}{|c|c|c|c|}
\hline Adjustment & $\begin{array}{l}\text { Cohort 1: all patients } \\
\quad(n=19,256)\end{array}$ & $\begin{array}{l}\text { Cohort 2: HDU-only patients } \\
\qquad(n=13,809)\end{array}$ & $\begin{array}{l}\text { Cohort 3: ICU patients } \\
\quad(n=5,447)\end{array}$ \\
\hline Unadjusted & $1.44(1.35,1.54), P<0.001$ & $1.38(1.26,1.51), P<0.001$ & $1.34(1.22,1.48), P<0.001$ \\
\hline Age & $1.42(1.33,1.51), P<0.001$ & $1.31(1.20,1.44), P<0.001$ & $1.28(1.16,1.41), P<0.001$ \\
\hline Age and sex & $1.40(1.31,1.49), P<0.001$ & $1.30(1.19,1.42), P<0.001$ & $1.28(1.16,1.41), P<0.001$ \\
\hline Age, sex, ethnicity & $1.36(1.27,1.45), P<0.001$ & $1.29(1.18,1.41), P<0.001$ & $1.23(1.11,1.36), P<0.001$ \\
\hline Age, sex, ethnicity, obesity & $1.32(1.23,1.41), P<0.001$ & $1.27(1.16,1.39), P<0.001$ & $1.29(1.17,1.43), P<0.001$ \\
\hline $\begin{array}{l}\text { Full covariate set adjustment model } \\
\text { (age, sex, ethnicity, obesity, } \\
\text { comorbidity*) }\end{array}$ & $1.23(1.14,1.32), P<0.001$ & $1.19(1.08,1.31), P<0.001$ & $1.24(1.11,1.38), P<0.001$ \\
\hline $\begin{array}{l}\text { Propensity score-matched model, } \\
\text { adjustment for full covariate set }\end{array}$ & $\begin{array}{c}1.25(1.14,1.36), P<0.001 \\
(n=3,289 \text { with diabetes } \\
\text { matched })\end{array}$ & $\begin{array}{c}1.17(1.04,1.32), P=0.01 \\
(n=2,007 \text { with diabetes } \\
\text { matched })\end{array}$ & $\begin{array}{c}1.19(1.05,1.35), P=0.009 \\
\text { ( } n=1,244 \text { with diabetes } \\
\text { matched })\end{array}$ \\
\hline $\begin{array}{l}\text { Competing risk model, adjustment for } \\
\text { full covariate set (Fine and Gray)** }\end{array}$ & $1.27(1.18,1.36), P<0.001$ & $1.24(1.13,1.36), P<0.001$ & $1.24(1.12,1.38), P<0.001$ \\
\hline
\end{tabular}

Age fitted as a restricted cubic spline with three knots in all models. ${ }^{*}$ Chronic respiratory disease, asthma, chronic heart disease, hypertension, immunosuppression, chronic neurological disease, chronic renal disease, chronic liver disease. **Fine and Gray model subdistribution hazard ratios taking into account the competing event of being discharged alive. 


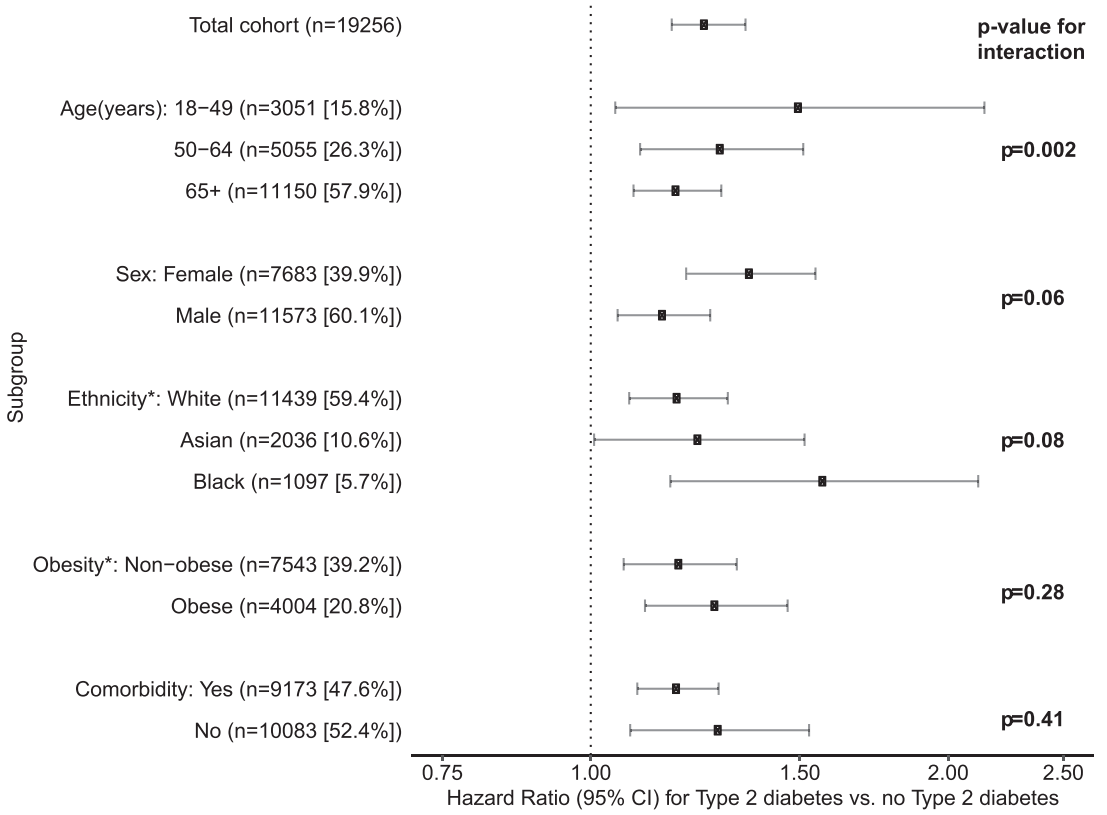

Figure 2-Major subgroup analysis of the primary outcome of 30-day in-hospital mortality. Hazard ratios represent the mortality risk associated with the presence of type 2 diabetes in each subgroup. Bars represent $95 \%$ Cls. *Hazard ratios for other subgroups: mixed ethnicity $1.91(95 \% \mathrm{Cl}$ $1.03,3.56)$, other ethnicity $0.96(0.67,1.39)$, missing ethnicity $1.34(1.11,1.62)$, missing obesity $1.28(1.08,1.46)$

males (i.e., no difference) and people of Asian ethnicity (i.e., increased risk) in critical care settings (Supplementary Table 2). The exception is that we did not find an association between obesity and mortality, in contrast to ICNARC, which reported an association of small magnitude for BMI but without adjustment for diabetes or hyperglycemia.

Previous studies of other viral respiratory infections suggest that findings of population-level studies should be extrapolated to the critical care setting with caution. For influenza, national surveys suggested increased influenza-specific mortality in people with diabetes (29); however, large multistate European studies of ICU admissions for influenza showed no difference in mortality by diabetes status (30). Our results, contrasted with the numerically much greater associations for diabetes reported in U.K. population-based studies of COVID-19 mortality, mirror this pattern of substantially reduced effect size $(7,8)$. Such setting-specific differences likely reflect different frames of reference, wherein each study is capturing a different portion of a complicated pathway including the risk of being infected and then accessing services, subsequently being admitted, hospital treatments, and finally, dying in hospital.
To our knowledge, CHESS is currently the largest critical care database of people with COVID-19 and coded diabetes status, providing near-complete capture of severe COVID-19 cases across England during the first wave of the pandemic (18). Our use of a methodologically robust modeling framework, with adjustment for potential confounders and multiple sensitivity analyses to evaluate the potential influence of missing data, lends additional credibility to our mortality estimates. Despite this, we cannot rule out unmeasured confounding as an explanation of our findings. Any interpretation of our results should be bounded by the knowledge that in the primary analysis we adjusted for variables along the causal pathway (e.g., cardiovascular comorbidities), which precludes any claim that associations relate to the causal role of type 2 diabetes in COVID-19 mortality (31). However, the consistent size of association for type 2 diabetes observed across models with sequential adjustment of clinical features supports the interpretation that type 2 diabetes is an important prognostic factor for critical care patients.

A limitation of the CHESS critical care data set is the lack of validated case definitions for recorded comorbidities, and it is possible that misclassification of diabetes status may have attenuated the mortality risk estimates observed in this study. A further important limitation is that the lack of standardized measurement of height and weight meant we had to adjust risk models for obesity status (BMI categorized as $<$ or $>30 \mathrm{~kg} / \mathrm{m}^{2}$ ) rather than with more granular categorization of BMI. A recent study of 6,916 U.S. patients diagnosed with COVID-19 suggests an independent J-shaped association between BMI category and mortality, with patients of $\mathrm{BMI}<18.5 \mathrm{~kg} / \mathrm{m}^{2}$ and $\geq 40 \mathrm{~kg} / \mathrm{m}^{2}$ at higher risk of mortality compared with those of normal BMI $\left(18.5-24 \mathrm{~kg} / \mathrm{m}^{2}\right)(32)$, and an analysis of 1,687 hospitalized adults in New York observed a similar J-shaped pattern between BMI and mortality, although with overweight patients at lowest risk (33). These analyses raise the possibility of residual confounding, due to the limited recording of $\mathrm{BMI}$ in CHESS, being a potential explanation of our findings. We lacked data to assess the impact of potentially modifiable diabetes-specific risk factors, in particular, hyperglycemia prior to, at, or during, hospital admission $(7,14,34-37)$, as well as possible modulatory effects of specific antihyperglycemic medications (38-40). Clinical information on duration of diabetes and the presence of diabetes complications was similarly not available. Robustly evaluating the added value of such potential diabetes-specific prognostic factors is an important area for ongoing $(35,37)$, and future, research to inform understanding of the mechanisms by which diabetes modifies outcome in severe COVID-19 (41), to establish which risk factors are most useful to identify which people with diabetes are most vulnerable to COVID-19 (42), and to inform individualized care strategies (38).

\section{Conclusion}

Type 2 diabetes may be an independent prognostic factor for in-hospital survival in people with severe COVID-19 admitted to a critical care setting. The additional mortality risk associated with type 2 diabetes is attenuated by age. These results can help inform in-hospital decisionmaking on appropriate care escalation and treatment provision for people with type 2 diabetes and severe COVID-19.

Acknowledgments. The authors thank PHE for providing access to CHESS data to the Warwick 
Research Group and Prof. Matt Keeling (University of Warwick) for his support and establishing data access.

Funding. This study was supported by Diabetes UK. J.M.D. is supported by an Independent Fellowship funded by Research England's Expanding Excellence in England (E3) fund. S.J.V., S.D., and B.A.M. are supported by The Alan Turing Institute (Engineering and Physical Sciences Research Council [EPSRC] grant EP/N510129/). R.S. receives a PhD stipend from EPRSC (EP/ R513143/1). S.J.V. is supported by the University of Warwick Impact Acceleration Account (IAA) funding. A.T.H. is a National Institute for Health Research (NIHR) Senior Investigator and a Wellcome Trust Senior Investigator (098395/Z/12/Z). J.M.D., N.J.T., K.A.P., A.T.H., and A.P.M. are supported by the NIHR Exeter Clinical Research Facility.

The funder had no role in the analysis or reporting of results.

Duality of Interest. A.P.M. declares previous research funding from Eli Lilly and Company, Pfizer, and AstraZeneca. S.J.V. declares funding from IQVIA. No other potential conflicts of interest relevant to this article were reported.

Author Contributions. J.M.D., B.A.M., N.J.T., A.P.M., and S.J.V. designed the study. J.M.D. and R.S. drafted code on dummy data. S.J.V. adapted and extended the code and executed on CHESS J.M.D., B.A.M., A.P.M., and S.J.V. drafted the manuscript. All authors provided support for the analysis and interpretation of results, critically revised the manuscript, and approved the final manuscript. J.M.D., B.A.M. and S.J.V. take responsibility for the integrity of the data and the accuracy of the data analysis. B.A.M. and S.J.V. are the guarantors of this work and, as such, had full access to all the data in the study and take responsibility for the integrity of the data and the accuracy of the data analysis.

\section{References}

1. World Health Organization. WHO DirectorGeneral's statement on IHR Emergency Committee on Novel Coronavirus (2019-nCoV). Accessed 16 October 2020. Available from https://www .who.int/dg/speeches/detail/who-director-general$\mathrm{s}$-statement-on-ihr-emergency-committee-on-novelcoronavirus-(2019-ncov)

2. Saeedi P, Petersohn I, Salpea P, et al.; IDF Diabetes Atlas Committee. Global and regional diabetes prevalence estimates for 2019 and projections for 2030 and 2045: results from the International Diabetes Federation Diabetes Atlas, 9th edition. Diabetes Res Clin Pract 2019; 157:107843

3. Zhou F, Yu T, Du R, et al. Clinical course and risk factors for mortality of adult inpatients with COVID-19 in Wuhan, China: a retrospective cohort study. Lancet 2020;395:1054-1062

4. Critchley JA, Carey IM, Harris T, DeWilde $S$, Hosking FJ, Cook DG. Glycemic control and risk of infections among people with type 1 or type 2 diabetes in a large primary care cohort study. Diabetes Care 2018;41:2127-2135

5. Shah BR, Hux JE. Quantifying the risk of infectious diseases for people with diabetes. Diabetes Care 2003;26:510-513

6. Magliano DJ, Harding JL, Cohen K, Huxley RR, Davis WA, Shaw JE. Excess risk of dying from infectious causes in those with type 1 and type 2 diabetes. Diabetes Care 2015;38:12741280

7. Williamson EJ, Walker AJ, Bhaskaran K, et al. Factors associated with COVID-19-related death using OpenSAFELY. Nature 2020;584: 430-436

8. Barron E, Bakhai C, Kar P, et al. Associations of type 1 and type 2 diabetes with COVID-19related mortality in England: a whole-population study. Lancet Diabetes Endocrinol 2020;8:813822

9. Li B, Yang J, Zhao F, et al. Prevalence and impact of cardiovascular metabolic diseases on COVID-19 in China. Clin Res Cardiol 2020;109: 531-538

10. Roncon L, Zuin M, Rigatelli G, Zuliani G. Diabetic patients with COVID-19 infection are at higher risk of $\mathrm{ICU}$ admission and poor short-term outcome. J Clin Virol 2020;127: 104354

11. Richardson S, Hirsch JS, Narasimhan M, et al.; Northwell COVID-19 Research Consortium. Presenting characteristics, comorbidities, and outcomes among 5700 patients hospitalized with COVID-19 in the New York City area. JAMA 2020 323:2052-2059

12. Docherty $A B$, Harrison EM, Green $C A$, et al.; ISARIC4C Investigators. Features of 20133 UK patients in hospital with covid-19 using the ISARIC WHO Clinical Characterisation Protocol: prospective observational cohort study. BM 2020;369:m1985

13. Shi $Q$, Zhang $X$, Jiang $F$, et al. Clinical characteristics and risk factors for mortality of COVID-19 patients with diabetes in Wuhan, China: a two-center, retrospective study. Diabetes Care 2020;43:13821391

14. Zhu L, She Z-G, Cheng X, et al. Association of blood glucose control and outcomes in patients with COVID-19 and pre-existing type 2 diabetes. Cell Metab 2020;31:1068-1077.e3

15. Yan $Y$, Yang $Y$, Wang $F$, et al. Clinical characteristics and outcomes of patients with severe covid-19 with diabetes. BMJ Open Diabetes Res Care 2020;8:e001343

16. Grasselli G, Greco M, Zanella A, et al. Risk factors associated with mortality among patients with COVID-19 in intensive care units in Lombardy, Italy. JAMA Intern Med 2020;180 1345-1355

17. Gupta S, Hayek SS, Wang W, et al.; STOPCOVID Investigators. Factors associated with death in critically ill patients with Coronavirus Disease 2019 in the US. JAMA Intern Med. 15 July 2020 [Epub ahead of print]. DOI: 10.1001/ jamainternmed.2020.3596

18. Public Health England. COVID-19 Hospitalisation in England Surveillance System (CHESS), 2020. Accessed 18 April 2020. Available from https://www.england.nhs.uk/coronavirus/wpcontent/uploads/sites/52/2020/03/phe-letterto-trusts-re-daily-covid-19-hospital-surveillance11-march-2020.pdf

19. Public Health England. COVID-19: investigation and initial clinical management of possible cases. 2020. Accessed 26 May 2020. Available from https://www.gov.uk/government/publications/ wuhan-novel-coronavirus-initial-investigationof-possible-cases/investigation-and-initialclinical-management-of-possible-cases-of-wuhannovel-coronavirus-wn-cov-infection
20. Groenwold RHH, White IR, Donders ART, Carpenter JR, Altman DG, Moons KGM. Missing covariate data in clinical research: when and when not to use the missing-indicator method for analysis. CMAJ 2012;184:1265-1269

21. Austin PC. A tutorial on multilevel survival analysis: methods, models and applications. Int Stat Rev 2017;85:185-203

22. Sterne JAC, White IR, Carlin JB, et al. Multiple imputation for missing data in epidemiological and clinical research: potential and pitfalls. BMJ 2009;338:b2393

23. $\mathrm{R}$ Development Core Team. $R$ : A Language and Environment for Statistical Computing. Vienna, Austria, R Foundation for Statistical Computing, 2011

24. Vlachos S, Wong A, Metaxa V, et al. Hospital mortality and resource implications of hospitalisation with COVID-19 in London, UK: a prospective cohort study. 7 July 2020 [preprint] medRxiv:2020.07.16.20155069

25. Mateen BA, Wilde H, Dennis JM, et al. A geotemporal survey of hospital bed saturation across England during the first wave of the COVID-19 Pandemic. 1 July 2020 [preprint]. medRxiv:2020.06.24.20139048

26. Qian Z, Alaa AM, van der Schaar M, Ercole A. Between-centre differences for COVID-19 ICU mortality from early data in England. Intensive Care Med 2020;46:1779-1780

27. Dennis J, McGovern A, Vollmer S, Mateen BA. Improving COVID-19 critical care mortality over time in England: a national cohort study, March to June 2020. 3 August 2020 [preprint]. medRxiv:2020.07.30.20165134

28. Intensive Care National Audit \& Research Centre (ICNARC). ICNARC report on COVID-19 in critical care, 2020. Accessed 15 August 2020. Available from https://www.icnarc.org/Our-Audit/ Audits/Cmp/Reports

29. Valdez R, Narayan KM, Geiss LS, Engelgau MM. Impact of diabetes mellitus on mortality associated with pneumonia and influenza among non-Hispanic black and white US adults. Am J Public Health 1999;89:1715-1721

30. Adlhoch C, Gomes Dias J, Bonmarin I, et al. Determinants of fatal outcome in patients admitted to intensive care units with influenza, European Union 2009-2017. Open Forum Infect Dis 2019;6:ofz462

31. Huitfeldt $A$. Is caviar a risk factor for being a millionaire? BMJ 2016;355:i6536

32. Coppelli A, Giannarelli R, Aragona M, et al. Hyperglycemia at hospital admission is associated with severity of the prognosis in patients hospitalized for COVID-19: the Pisa COVID-19 Study. Diabetes Care 2020;43:2345-2348

33. Holman N, Knighton $P$, Kar $P$, et al. Risk factors for COVID-19-related mortality in people with type 1 and type 2 diabetes in England: a population-based cohort study. Lancet Diabetes Endocrinol 2020;8:823-833

34. Riddle MC. Diabetes and COVID-19: moving from news to knowledge and a glucose hypothesis. Diabetes Care 2020;43:2336-2338

35. Cariou B, Hadjadj S, Wargny M, et al.; CORONADO investigators. Phenotypic characteristics and prognosis of inpatients with COVID-19 and diabetes: the CORONADO study. Diabetologia 2020;63:1500-1515

36. Coppelli A, Giannarelli R, Aragona M, et al.; Pisa COVID-19 Study Group. Hyperglycemia at hospital 
admission is associated with severity of the prognosis in patients hospitalized for COVID-19: the Pisa COVID19 study. Diabetes Care 2020;43:2345-2348

37. Holman N, Knighton $P$, Kar $P$, et al. Risk factors for COVID-19-related mortality in people with type 1 and type 2 diabetes in England: a population-based cohort study. Lancet Diabetes Endocrinol 2020;8:823-833

38. Scheen AJ, Marre M, Thivolet C. Prognostic factors in patients with diabetes hospitalized for
COVID-19: findings from the CORONADO study and other recent reports. Diabetes Metab 2020; 46:265-271

39. Apicella M, Campopiano MC, Mantuano M, Mazoni L, Coppelli A, Del Prato S. COVID-19 in people with diabetes: understanding the reasons for worse outcomes. Lancet Diabetes Endocrinol 2020;8:782-792

40. Chen Y, Yang D, Cheng B, et al. Clinical char acteristics and outcomes of patients with diabetes and COVID-19 in association with glucose-lowering medication. Diabetes Care 2020;43:1399-1407 41. Mauvais-Jarvis F. Aging, male sex, obesity, and metabolic inflammation create the perfect storm for COVID-19. Diabetes 2020;69:18571863

42. Riddle MC, Buse JB, Franks PW, et al. COVID-19 in people with diabetes: urgently needed lessons from early reports. Diabetes Care 2020;43:1378-1381 